Outcome des Patienten und senkt die Letalitätsrate. Bei Instabilität des Kreislaufs wird, in Abschätzung etwaiger Vorerkrankungen, zunächst mit einer gesteigerten Volumensubstitution gegengesteuert. Das optimale Zeitfenster $>90$ Minuten bestimmt auch hier die Prognose des Patienten.

Kristalloide Lösungen sind kolloiden Lösungen vorzuziehen. Studien zufolge hat die Infusionstherapie unter synthetisch-hergestellten Kolloidlösungen (z.B. HAES) zu stärkerer Schädigung der Nierenfunktion geführt. Die Patienten mussten öfter hämodialysiert werden, verblieben länger im Krankenhaus und hatten eine schlechtere Prognose. Im Einzelfall könnte daher die Gabe von Humanalbumin oder Gelantine erwogen werden. Bleibt der Patient im Verlauf hypotensiv, wird die Therapie häu- fig mit Noradrenalin erweitert. Reicht die Infusionstherapie nicht aus (arterieller Mitteldruck $\leq 65$, schlechte Diurese usw.), um das Herzeitvolumen zu steigern, wird Dobutamin als Katecholamin der ersten Wahl empfohlen.

\section{Glukokortikosteroide und Insulintherapie}

Die Behandlung mit hochdosierten Glukokortikosteroiden wird in der Therapie mit schwerer Sepsis und septischem Schock nicht empfohlen, in niedriger Dosis nur als letztes Mittel der Wahl. Eine moderate Insulintherapie ist einer intensivierten Insulintherapie vorzuziehen, um das Risiko schwerer Hypoglykämien zu vermeiden.

Während die möglichst schnelle Fokussanierung, Antibiotikagabe und Kreislaufstabilisierung zum

\title{
SEPSIS STEIGERT THROMBOSERISIKO
}

Patienten mit einer präoperativen Sepsis sind besonders gefährdet, nach dem Eingriff eine arterielle oder venöse Thrombose zu erleiden. Das Risiko steigt mit dem Schweregrad der systemischen Entzündung.

Eine Sepsis ist bei Op.-Kandidaten ein wichtiger unabhängiger Risikofaktor für postoperative Thrombosen: Ihr Risiko für einen arteriellen oder venösen Gefäßverschluss ist dreimal so hoch wie das von Patienten ohne systemische Entzündung. Zu diesem Ergebnis kommen Ärzte um Jacques D. Donzé vom Inselspital Bern, die 2,3 Millionen Patientenakten der Jahre 2005-12 aus dem US-amerikanischen Chirurgieregister ACS-NSQIP ausgewertet haben. Bei 167.986 Patienten war in den 48 Stunden vor dem Eingriff eine Sepsis diagnostiziert worden.

Thrombosen traten in den 30 Tagen nach einer Op. umso häufiger auf, je gravierender eine präoperative Sepsis war. Bestand nur ein SIRS - ohne Nachweis einer Infektion - war das Risiko um den Faktor 2,5, bei einer Sepsis um den Faktor 3,3 und bei einer schweren Sepsis um den Faktor 5,7 erhöht.

Thrombosen der venösen Strombahn wurde etwas stärker gefördert. Die Wahrscheinlichkeit für eine tiefe Venenthrombose oder Lungenembolie war mit Sepsis 3,3-mal so hoch wie ohne, die für Herzinfarkt oder Schlaganfall 2,7-mal. Bei Sepsispatienten waren sowohl notfallmäßige als auch elektive Operationen mit einem erhöhten Thromboserisiko verbunden; Notfalloperationen erwiesen sich nur im Hinblick auf venöse Thromboembolien als gefährlicher.

Der Zusammenhang zwischen Sepsis und Thrombose bestand unabhängig vom Vorliegen einer Krebserkrankung oder eines Diabetes. Die sepsis- bedingten Risikosteigerungen fielen allerdings bei Patienten ohne Krebs bzw. ohne Diabetes höher aus.

"Unsere Ergebnisse sind klinisch einleuchtend", schreiben Donzé und Kollegen. Da eine Sepsis mit Entzündung und einem Zustand der Hyperkoagulabilität einhergehe, entspreche das vermehrte Auftreten von Thrombosen den Erwartungen. Die Ärzte empfehlen daher, in die Nutzen-RisikoBewertung eines chirurgischen Eingriffs ,jeden Schweregerad einer Sepsis als Risikofaktor sowohl für arterielle als auch für venöse Thrombosen" einzubeziehen.

Das Risiko für Herzinfarkt oder Schlaganfall werde durch eine Sepsis in ähnlichem Maße erhöht wie etwa durch eine Herzinsuffizienz oder eine zerebrovaskuläre Erkrankung. „Im Gegensatz zu Letzteren kann aber noch mehr unternommen werden, um das kardiovaskuläre Risiko von Sepsispatienten zu senken", so die Studienautoren. Das könne eine Verbesserung der kardiovaskulären Protektion sein, zum Beispiel durch Statine. Vor allem aber müsse eine Operation, wann immer möglich, so lange verschoben werden, bis die Sepsis behandelt sei. Eine verbesserte Vorbeugung halten die Autoren auch im Hinblick auf venöse Thromboembolien für angezeigt: „Viele chirurgische Patienten mit einer Sepsis könnten von einer Thromboseprophylaxe profitieren."

Donzé JD et al. Impact of sepsis on risk of postoperative arterial and venous thromboses: large prospective cohort study. BMJ 2014; 349: g5334 (doi: 10.1136/bmj.g5334)
Der Zeitpunkt der Diagnose und eine umgehende Initiierung der Behandlung sind entscheidend für das Überleben eines septischen Patienten.

Ein speziell für Sepsis zugelassenes Medikament gibt es derzeit nicht. 